\title{
Smart Blind Stick Design and Implementation
}

\author{
Amira. A. Elsonbaty
}

\begin{abstract}
Technologies are rapidly evolving, allowing people to live healthier and simpler lives. Sightless people are unable to carry out their everyday activities, such as walking down the street, visiting friends or relatives, or doing some other mundane tasks. As a result, the smart stick is a stick that can assist a person in walking safely without fear of colliding with another person or solid objects is proposed as a solution to this major issue. It is a development of the traditional blind stick as it acts as a companion for the blind when walking by sending audio alerts to the blind via a headphone connected to the phone with obstacles (water/walls/stairs / muddy ground) and also enables him to make a phone call to ask for help. EasyEdasoftware was used for designing and simulating electrical circuits, was used to model the electric circuit.This system functions similarly to a white cane in that it assists blind people in scanning their surroundings for obstacles or orientation marks. This system will be mounted on a white cane with an ultrasonic sensor, and a water sensor to detect changes in the environment. Ultrasonic sensors detect obstacles in front of it using ultrasonic wave reflection, water detection sensors detect whether there is a puddle.
\end{abstract} Android.

Keywords: Blind Stick, SmartSystems, Embedded Systems.

\section{INTRODUCTION}

B lindness is a state of lack of visual perception due to physiological or neurological factors. Imagine that you are walking in an unfamiliar place[1]. One has to ask for guidance to get to the destination. But what if the person is visually impaired. A person must completely depend on other people to get to the destination. In general, we note that the white cane is the best friend of visually impaired people. But oftentimes that stick isn't helpful. The Blind Stick is developed using many hardware and software applications.An individual with a disability is a member of society and has the same rights and responsibilities as people. But blind people face a large number of problems that are difficult to solve. Blind people are members of society, and their diversity in the world and social situations has been restricted. Blind people's disadvantages should not be seen as an excuse to shorten their lives; rather, they should be used as motivation to persevere. As a result, anyone with visual impairments requires assistance in the form of replacements for their eye function, specifically the visual function. In addition to the normal touch sticks, the blind often needs a switch for their sense of sight so that the ultrasonic and sound sensors can be used.

Manuscript received on April 23, 2021.

Revised Manuscript received on April 30, 2021.

Manuscript published on June 30, 2021.

* Correspondence Author

Amira. A. Elsonbaty*, Communication \& Electronics Department, Higher institute of engineering and technology, New Damietta, Egypt, 34517 Email: Amira.elsonbaty@ndeti.edu.eg

(c) The Authors. Published by Blue Eyes Intelligence Engineering and Sciences Publication (BEIESP). This is an open access article under the CC BY-NC-ND license (http://creativecommons.org/licenses/by-nc-nd/4.0/)
This smart stick will warn blind people about obstacles using the audio jack headphones, allowing the blind person to avoid an object in front of them[2]

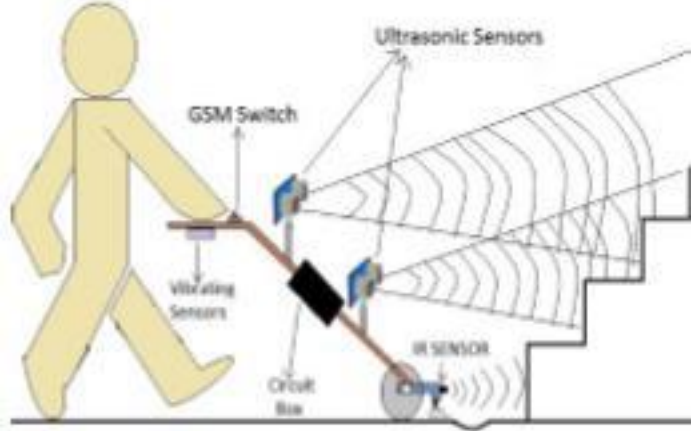

Fig.1. :Blind Man with Electronic Stick

\section{PRINCIPLE OF OPERATION}

The System Development Life Cycle (SDLC) is the process of developing and changing processes, as well as the models and methodologies used to construct an application and a software development process[3]. It involves the following steps:

A. Preparation: Needs evaluations, feasibility studies (both scientific and technological), and scheduling are also carried out as part of the planning phase.

B. Analysis:Direct observation is used during the research process to look at the problems that arise and are found in the materials, software, and hardware.

C. Design: At this point, the application will be explained in detail regarding the design phase of each component in the prototype under the needs addressed earlier in the prototype[4].

D. Implementation:The code is brought to life at this stage by selecting components and planning the software (coding/coding).

E. Testing:Testing is carried out at this point to see if the framework created satisfies the user's needs; if it does not, the next phase is iterative, i.e. returning to the previous stages. And the test is designed to identify and eliminate flaws in the device so that it can truly assist users in their everyday activities.

F. Maintenance:The system's operation starts at this stage, and minor repairs can be made if necessary.

\section{PRIMARY OBJECTIVES}

The main aim is to make new technology easier to use for visually disabled people. In a technology-controlled world where people strive to live independently, this work proposes an ultrasonic stick for

blind people to aid them in achieving personal independence. It is simple to use due to its low cost and lack of bulk.

Published By: Sciences Publication

(C) Copyright: All rights reserved.

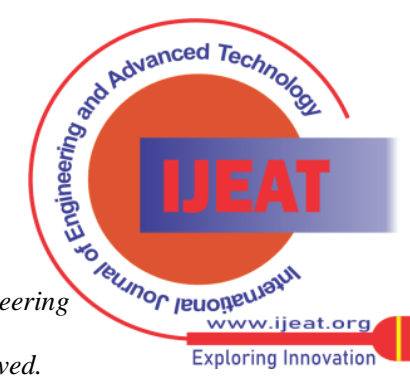


The ultrasonic sensor senses the presence of an obstruction and calculates the distance between the source and the target, a water sensor is used to detect the presence of water, and a fire sensor is used to detect the presence of fire by an alarm, vibration and sound.

\section{PROPOSED SYSTEM}

During the evaluation of this method, opinions on individuals with visual impairments were formed through discussions and interviews with users to identify resources designed for blind people with disabilities. The Design of a Stick Prototype for People with Visual Impairment Using Ultrasonic Esp8266 is discussed below. The views of blind people are one of the subjects addressed to receive feedback/appraisal from users. Three users were given the task of rating the prototype, as well as trying it out and responding to the statements made.

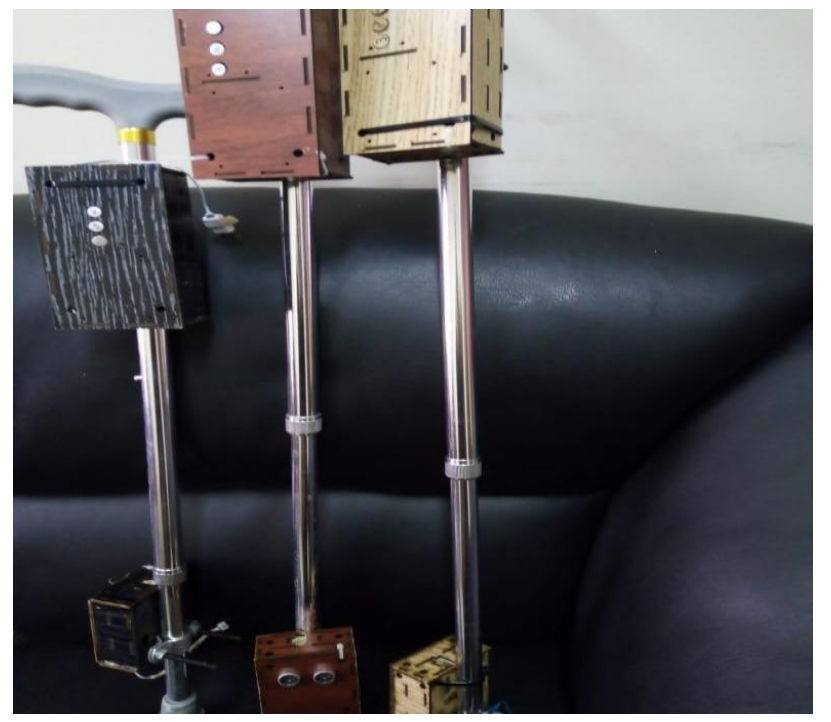

Fig.2. Three Experimental Sticks

Based on the findings, it can be inferred that ultrasonic sensor sticks have proven to be extremely beneficial to blind people. This prototype has reduced the risk of blind people getting into accidents in difficult road structures with many obstacles, as well as when crossing the street. Since a large gap between the stick and the sensor can result in constant censorship of objects recognized around the stick, the design of the blind stick is made more flexible on the stick section, which is something to consider. The stick works by creating an Android-based mobile application that links the stick to the phone and performs a variety of tasks, including making phone calls to pre-determined numbers and determining the location. The stick is distinguished by its low price and simple nature. When the wireless sensor detects an object or obstacle in its environment, it serves as an input or input to the esp8266 processor. The audio jack connected to the headphone then emits sound. The lack of essential skills and preparation, as well as the limited range of motion and knowledge transmitted, are among the most serious shortcomings of these aids. Electronic assistive devices are intended to solve issues like these, and we used some electronics modules and sensors to adjust the cane. A buzzer, ultrasonic sensors, and a water sensor are all included.The blind person walking with an electronic stick. Two ultrasonic sensors are mounted on the stick having a set to different rangesfor avoiding small obstacles. Three push buttons that can be operated with the thumballows the blind user to send a general message (I am in trouble, help me) on a saved mobile no, or make a call for help. Vibrating sensors along with a buzzer used for beep and vibration if the stick is about to hit any obstacle. The circuit box containsa combination of microcontroller circuitry. The co-operation between the Ultrasonic and others sensors are utilized to create a complementary system that can give reliable distance measurement.A schematic circuit is made to make it easier to make tools. The schematic consists of Esp8266, Battery, Ultrasonic Sensor, water sensor. The ultrasonic sensors in our proposed project are used to detect obstacles ahead using ultrasonic waves. When the sensor detects obstacles, it sends the information to the Esp8266. The Esp8266 then analyzes the information and determines if the obstacle is near enough. If the obstacle isn't near enough, the circuit has no impact. If the obstacle is approaching, the Esp8266 issues a voice warning. It also senses water and warns the blind by sounding. The vibrator is also included in the stick. If the obstacle is approaching, the Esp8266 vibrates to alert you. A water sensor is used to sense water.

\section{SYSTEM DESCRIPTION}

\section{A. Ultrasonic Sensor}

It is an ultrasonic sensor, also known as an ultrasonic transducer, that is based on a transmitter and receiver and is primarily used to determine the distance from a target object with a wavelength ranging from $20 \mathrm{kHz}$ to $20 \mathrm{MHz}$ [5].

Ultrasonic sensors, like sonar detectors, work by transmitting a pulse of sound outside the range of human hearing. At the speed of sound $(340 \mathrm{~m} / \mathrm{s})$, this pulse travels away from the range finder in a conical shape. The sound bounces off an object and is reflected in the range finder. This is interpreted as an echo by the sensor, which measures the time between transmitting the signal and receiving the echo. The object's distance is then calculated using this interval by a controller in simple notation[6]:

$$
\text { distance }=\frac{\text { elapsed time } x \text { speed of sound }}{2}
$$

The ultrasonic sensor is a robust and flexible sensing agent with relatively few limitations.Our ultrasonic sensors are in the air, non-contact object detection and ranging sensors that detect objects within an area. These

sensors are not affected by the colour or other visual characteristics of the detected object. Ultrasonicsensors use high-frequency sound to detect and localize objects in a variety of environments. Ultrasonic sensors measure the time of flightfor the sound that has been transmitted to and reflected from nearby objects. Based upon the time of flight, the sensorthen outputs a range reading.

Blue Eyes Intelligence Engineering and Sciences Publication (C) Copyright: All rights reserved.

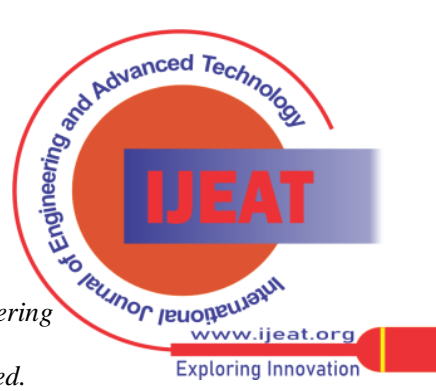




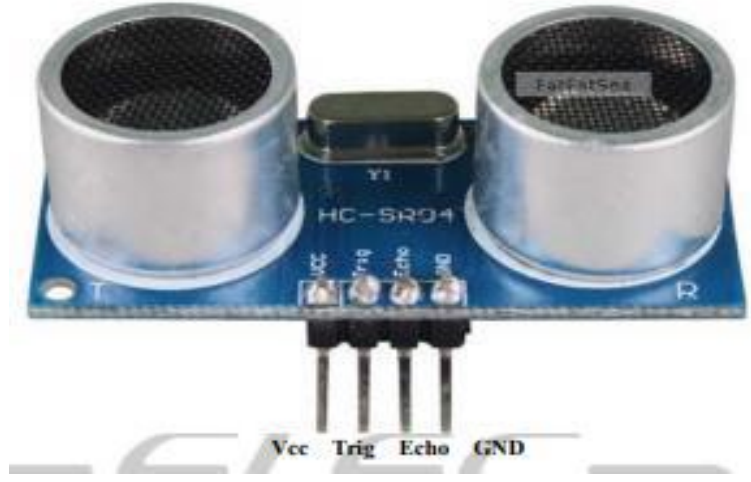

Fig3: Ultrasonic

\section{B.NodeMCU ESP8266}

The NodeMCU ESP8266 development board comes with the ESP-12E module containing the ESP8266 chip having TensilicaXtensa 32-bit LX106 RISC microprocessor. This microprocessor supports RTOS and operates at $80 \mathrm{MHz}$ to $160 \mathrm{MHz}$ adjustable clock frequency. NodeMCU has 128 $\mathrm{KB}$ RAM and $4 \mathrm{MB}$ of Flash memory to store data and programs. Its high processing power with in-built Wi-Fi / Bluetooth and Deep Sleep Operating features make it ideal for IoT projects[7]. The NodeMCU Development Board can be easily programmed with Arduino IDE since it is easy to use.

\section{Water Sensor}

Water sensor brick is designed to detect water and can be used to detect rainfall, water level, and even liquid leakage. An Electronic brick connector, a $1 \mathrm{M}$ resistor, and many lines of bare conducting wires make up the majority of the brick[8]

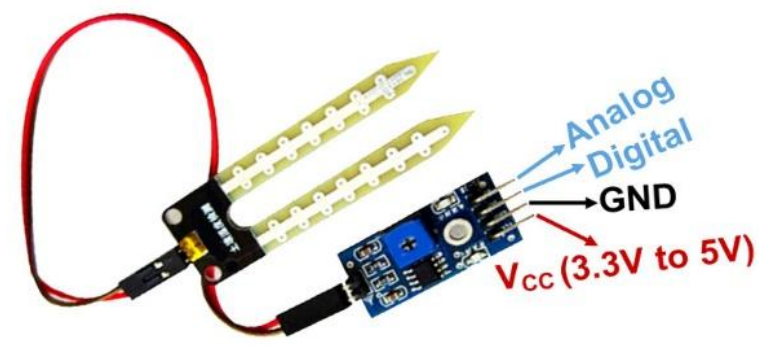

Fig.4.water sensor

\section{PROTOTYPE}

The smart white cane is design as shown in fig 4,5 the stick uses ultrasonic sensors mounted on the stick sothat it will assist in carrying out daily activities, the water detector is placed at the front end of the stick which aims to detect whether theroad to be inundated by water or not and it connected to the speaker/buzzer.

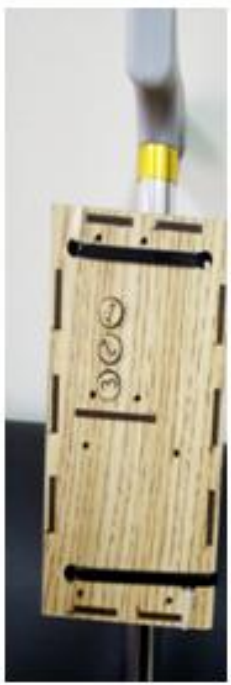

Fig.5.a.Three push boutton

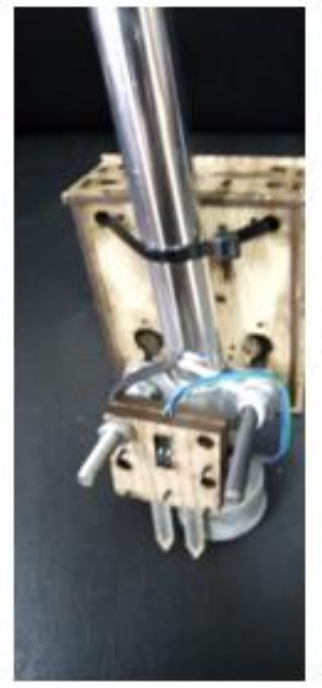

Fig.5.b.

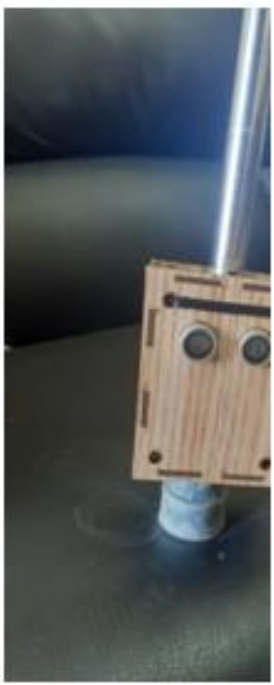

Fig.5.a.Ultrasonic Box
Fig.5. The final result

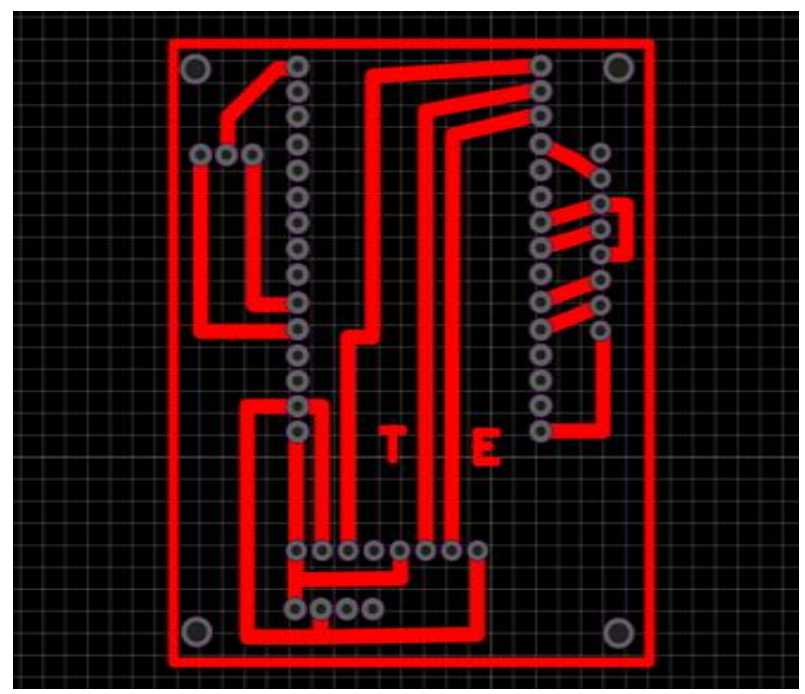

Fig.6. circuits by easy eda software

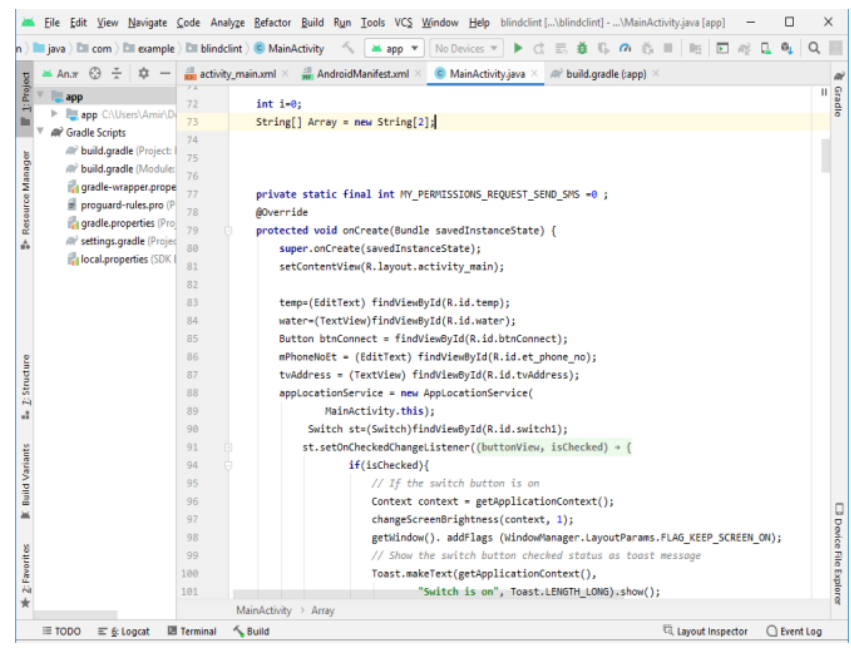

Fig.7. the shot screen of android code

Published By: Blue Eyes Intelligence Engineering and Sciences Publication (C) Copyright: All rights reserved.

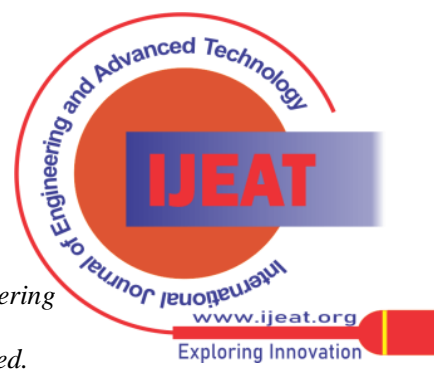




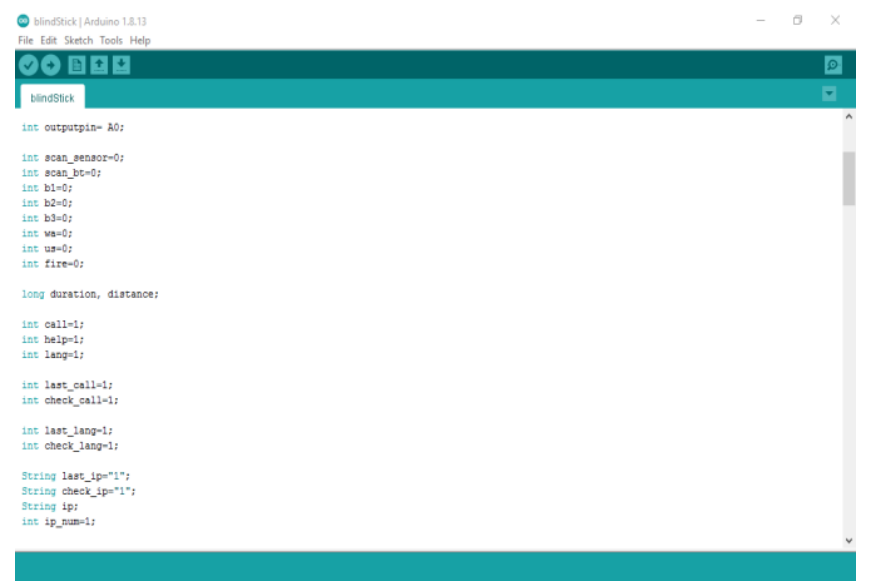

Fig.8. the shot screen of Arduino ide

\section{ADVANTAGES\& DISADVANTAGES}

\section{A. Advantages}

1) Both indoor and outdoor navigation are possible with the device.

2) The location of a blind person may be monitored at any time, providing added security.

3) Detects obstacles and notifies the blind person through vibration and speech production.

\section{B. Disadvantages}

1) The battery must be charged.

2) If the stick is not charged, it will not work.

\section{CONCLUSION}

This paper has created a concept prototype of sticks for blind people that uses sensor technology to aid alertness and movement in the blind. The blind can sense objects up to 70 centimetres away and receive feedback in the form of sound and vibration. At this point, it's worth noting that the study's primary objective, which was to create and launch a smart walking stick for the blind, was fully achieved. The Smart Stick is a building block for the next generation of assistive technology that can help the visually impaired navigate both indoor and outdoor environments safely. It is both effective and powerful. Within a three-meter range, it performs well in detecting obstacles in the user's direction. With a noticeable short response time, this system provides a lowcost, dependable, lightweight, low-power, and robust navigation solution. Despite being hard-wired with sensors and other parts, the system is lightweight. Ultrasonic sensors with a large beam angle can detect a wide range of obstacles. In the future, further improvements will be made to enhance the system's efficiency. Both include a global positioning system (GPS) for determining the user's location and GSM modules for informing a parent or caretaker of the user's location. For ease of use, it should be able to handle a variety of grips.

\section{REFERENCES}

1. S Romadhon, A K Husein," Smart Stick for the Blind Using Arduino", International Conference on Science and Technology 2019, doi:10.1088/1742-6596/1569/3/032088, 2019.

2. Shubham Bele , Swapnil Ghule , AkshayGunjal , N.D. Anwat," Design and Implementation of Smart Blind Stick", International Conference on Communication and Information Processing (ICCIP2020), 2020.

Published By:

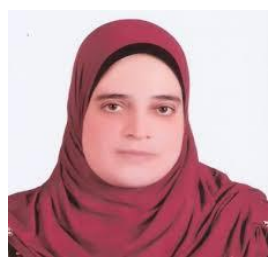

Amira. A. Elsonbaty, Lecturer and AcademyCoordinator in Higher Institute of engineering \&technology in new DamiettaFields: Smart building management- Internet of thingsArtificial intelligenceNeural networks- Robotics engineering - Communication networks \& Digital communication- Wireless sensor networksGreen cloud computing Software-Defined Networking - Control systems over wireless networks- publications: forest fires detection using machine learning techniques- Arabic handwritten character recognition based on convolution neural networks and support vector machine- the smart parking management system- robot navigation using a neural network extracts from the decision tree- smart college using the internet of things- survey of cloud computing
Blue Eyes Intelligence Engineering and Sciences Publication (C) Copyright: All rights reserved.

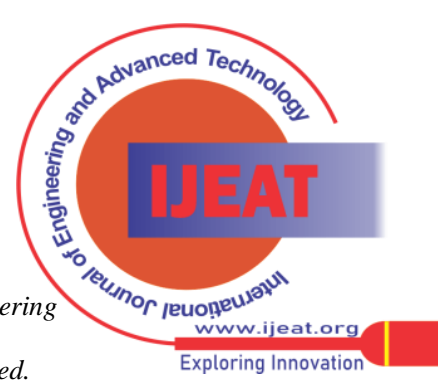

